\title{
Ghostbursting: the effects of dendrites on spike patterns
}

\author{
Carlo R. Laing ${ }^{\mathrm{a}, *}$, Brent Doiron ${ }^{\mathrm{a}, \mathrm{b}}$, André Longtin ${ }^{\mathrm{a}}$, Len Maler ${ }^{\mathrm{b}}$ \\ ${ }^{a}$ Department of Physics, University of Ottawa, 150 Louis Pasteur, Ottawa, Ont., Canada K1N 6N5 \\ ${ }^{\mathrm{b}}$ Department of Cellular and Molecular Medicine, University of Ottawa, 451 Smyth Road, \\ Ottawa, Ont., Canada K1H $8 \mathrm{M} 5$
}

\begin{abstract}
We present a two-compartment model of a pyramidal cell from the electrosensory lateral line lobe of weakly electric fish. These cells undergo a complex form of bursting, previously labeled "ghostbursting", and our model reproduces many aspects of experimental recordings. We analyze the effects of varying both the conductance between the somatic and dendritic compartments in the model, and the ratio of somatic to whole-cell areas, and find that both must have moderate values for bursting to occur. This is explained in terms of the previously elucidated burst mechanism. (c) 2002 Published by Elsevier Science B.V.
\end{abstract}

Keywords: Bursting; Pyramidal cell; Bifurcation; Compartmental model

\section{Introduction}

Recently, a novel bursting mechanism was identified in pyramidal cells in the electrosensory lateral line lobe (ELL) of the gymnotiform weakly electric fish Apteronotnus leptorhynchus $[2,3,10,14]$. Experimental in vitro recordings from these cells $[10,14]$ show that under constant current injection they fire regular bursts of action potentials. One of the most striking aspects of these bursts is that the interspike intervals (ISIs) decrease monotonically during a burst, i.e. the instantaneous frequency (reciprocal of the current ISI) increases monotonically through the burst, and the burst ends with a high-frequency doublet. This contrasts with many other types of bursting cells [8], in which theoretical analysis [7] shows that ISIs either increase towards the end of a burst or show no significant trend either way.

\footnotetext{
* Corresponding author. Tel.: +1-613-562-5800x6744; fax: +1-613-562-5190.

E-mail addresses: claing@science.uottawa.ca (C.R. Laing), bdoiron@physics.uottawa.ca (B. Doiron), alongtin@physics.uottawa.ca (A. Longtin), lmaler@aix1.uottawa.ca (L. Maler).
} 
Biophysically based multicompartmental models [2,3] have been successful in reproducing these observed bursts, and with the aid of experimental results $[10,14]$, the mechanism involved in bursting has been understood. In summary, the dendrite and soma of the neuron are both capable of producing action potentials, but the refractory period of the dendrite is longer than that of the soma. During a burst, the dendritic action potentials (which follow the somatic ones via backpropagation) effectively provide a weak positive feedback to the soma which results in depolarizing afterpotentials (DAPs) at the soma, and the sizes of these DAPs increase on a slow time scale, due to slow inactivation of dendritic $\mathrm{K}^{+}$channels - this is responsible for the increasing instantaneous frequency at the soma. The burst terminates when a somatic ISI is smaller than the refractory period of the dendrite, so the dendrite no longer fires an action potential in response to one at the soma, and the effect of the positive feedback is rapidly removed, producing a long ISI that groups spike clusters into bursts. The burst mechanism (labeled "ghostbursting") is explained in greater detail in Refs. [2,3,10,14].

One consequence of this form of bursting is that if the magnitude of a DC current injected to a pyramidal cell is slowly increased, the cell changes from quiescent to tonic (periodic) firing of action potentials to bursting. The two bifurcations separating the three types of behavior were determined in Ref. [2] to be a saddle-node bifurcation of fixed points on a circle, and a saddle-node bifurcation of periodic orbits, respectively. This sequence is in contrast to many other burst mechanisms, where the sequence is quiescent $\rightarrow$ bursting $\rightarrow$ tonic firing, as applied current is increased. The "burst threshold" in the ghostburster is demonstrated experimentally in Ref. [10] and discussed further in Ref. [2]. This threshold is very important if these neurons are involved in feature detection [6] and information processing [11], since information from other cells will be manifested as a change in input current to a pyramidal cell, which may then cause a change from periodic firing to bursting or vice versa.

In Ref. [3], a multicompartment model of an ELL pyramidal cell was presented, and in Ref. [2] a simplified version of this model was analyzed. Bifurcation analysis was done using the injected current to the soma and the dendritic potassium conductance as parameters. In this paper, we extend the bifurcation analysis using (a) the soma to dendrite coupling conductance, and (b) the ratio of the somatic area to the area of the whole cell, as parameters.

\section{Model}

The model consists of two isopotential compartments, representing the soma and dendrite of the neuron. They are diffusively coupled through voltage, following Refs. $[12,13]$. The equations, previously presented in Ref. [2], are

$$
\begin{aligned}
C \frac{\mathrm{d} V_{\mathrm{s}}}{\mathrm{d} t}= & I-g_{\mathrm{Na}, \mathrm{s}}\left[m_{\infty, \mathrm{s}}\left(V_{\mathrm{s}}\right)\right]^{2}\left(h_{0}-n_{\mathrm{s}}\right)\left(V_{\mathrm{s}}-V_{\mathrm{Na}}\right)-g_{\mathrm{K}, \mathrm{s}} n_{\mathrm{s}}^{2}\left(V_{\mathrm{s}}-V_{\mathrm{K}}\right) \\
& -g_{L}\left(V_{\mathrm{s}}-V_{L}\right)-\frac{g_{\mathrm{c}}}{\kappa}\left(V_{\mathrm{s}}-V_{\mathrm{d}}\right),
\end{aligned}
$$




$$
\begin{aligned}
\frac{\mathrm{d} n_{\mathrm{s}}}{\mathrm{d} t}= & \frac{n_{\infty, \mathrm{s}}\left(V_{\mathrm{s}}\right)-n_{\mathrm{s}}}{0.39}, \\
C \frac{\mathrm{d} V_{\mathrm{d}}}{\mathrm{d} t}= & -g_{\mathrm{Na}, \mathrm{d}}\left[m_{\infty, \mathrm{d}}\left(V_{\mathrm{d}}\right)\right]^{2} h_{\mathrm{d}}\left(V_{\mathrm{d}}-V_{\mathrm{Na}}\right)-g_{\mathrm{K}, \mathrm{d}} n_{\mathrm{d}}^{2} p_{\mathrm{d}}\left(V_{\mathrm{d}}-V_{\mathrm{K}}\right) \\
& \quad-g_{L}\left(V_{\mathrm{d}}-V_{L}\right)-\frac{g_{\mathrm{c}}}{1-\kappa}\left(V_{\mathrm{d}}-V_{\mathrm{s}}\right), \\
\frac{\mathrm{d} h_{\mathrm{d}}}{\mathrm{d} t}= & h_{\infty, \mathrm{d}}\left(V_{\mathrm{d}}\right)-h_{\mathrm{d}}, \\
\frac{\mathrm{d} n_{\mathrm{d}}}{\mathrm{d} t}= & \frac{n_{\infty, \mathrm{d}}\left(V_{\mathrm{d}}\right)-n_{\mathrm{d}}}{0.9}, \\
\frac{\mathrm{d} p_{\mathrm{d}}}{\mathrm{d} t}= & \frac{p_{\infty, \mathrm{d}}\left(V_{\mathrm{d}}\right)-p_{\mathrm{d}}}{5} .
\end{aligned}
$$

Subscripts $\mathrm{s}$ and $\mathrm{d}$ refer to somatic and dendritic variables, respectively. $m$ and $h$ are activation and inactivation of $\mathrm{Na}^{+}$, respectively, and $n$ and $p$ are activation and inactivation of $\mathrm{K}^{+}$, respectively. The parameter values used are $C=1, g_{\mathrm{Na}, \mathrm{s}}=55, h_{0}=1$, $V_{\mathrm{Na}}=40, g_{\mathrm{K}, \mathrm{s}}=20, V_{\mathrm{K}}=-88.5, g_{\mathrm{L}}=0.18, V_{L}=-70, g_{\mathrm{c}}=1.0, \kappa=0.4, g_{\mathrm{Na}, \mathrm{d}}=$ $5, g_{\mathrm{K}, \mathrm{d}}=15 . I$ is the input current, $g_{\mathrm{c}}$ is the coupling conductance, and $\kappa$ is the ratio of the somatic area to the total area of the cell. $g_{\mathrm{c}}$ and $\kappa$ are varied below. Other functions are $m_{\infty, \mathrm{s}}(V)=1 /[1+\exp (-(V+40) / 3)], n_{\infty, \mathrm{s}}(V)=1 /[1+\exp (-(V+40) / 3)]$, $m_{\infty, \mathrm{d}}(V)=1 /[1+\exp (-(V+40) / 5)], h_{\infty, \mathrm{d}}(V)=1 /[1+\exp ((V+52) / 5)], n_{\infty, \mathrm{d}}(V)=$ $1 /[1+\exp (-(V+40) / 5)], p_{\infty, \mathrm{d}}(V)=1 /[1+\exp ((V+65) / 6)]$. For details and derivation of these equations, see Refs. [2,3].

In Ref. [2], we investigated the behavior of system (1)-(6) as both $I$ and $g_{\mathrm{K}, \mathrm{d}}$ were varied, and showed that if $g_{\mathrm{K}, \mathrm{d}}$ was decreased, the curve of saddle-node bifurcations of fixed points (separating quiescent from tonic behavior) and the curve of saddle-node bifurcations of periodic orbits (separating tonic firing from bursting) could be brought together at a codimension-two point. In this paper, we investigate the effects of varying $\kappa$ and $g_{\mathrm{c}}$ (and $I$ ), as was done in Refs. $[5,9,12]$, where they were shown to have strong effects on firing patterns.

\section{Results}

\subsection{Varying $\kappa$ (somatic to total area ratio)}

In Fig. 1, we show the partial bifurcation set using the applied current, $I$, and the ratio of the somatic area to the total area, $\kappa$, as parameters. Previously [2] we set $\kappa=0.4$. For a given $\kappa>\sim 0.35$, as $I$ is increased the cell starts to fire periodically as a result of the saddle-node bifurcation of fixed points on a circle (solid line). For $\kappa$ between $\sim 0.35$ and $\sim 0.5$, this periodic firing ends due to a saddle-node bifurcation of periodic orbits (dashed line), leading to (often chaotic) bursting. For $\kappa>0.5$, only periodic firing occurs, and for $\kappa<0.35$, the neuron moves from quiescence to doublet firing as $I$ is increased. 


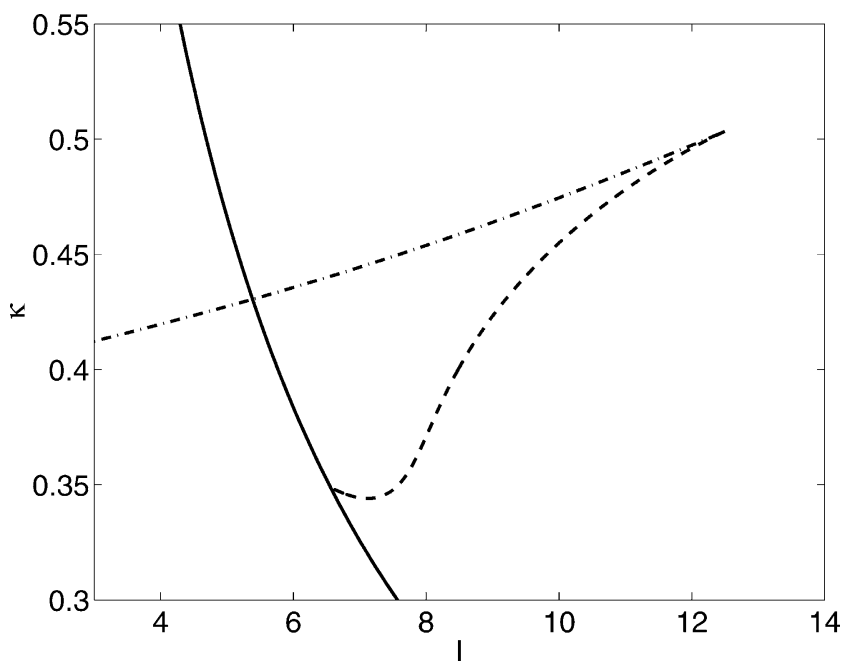

Fig. 1. Bifurcation set using $I$ and $\kappa$ as bifurcation parameters. The solid curve indicates a saddle-node bifurcation of fixed points on a circle, dashed is a saddle-node bifurcation of periodic orbits (one of which is stable) marking the tonic to burst transition, and the dashed-dotted is a saddle-node bifurcation of periodic orbits, both of which are unstable. $g_{\mathrm{c}}=1$.

The behavior in Fig. 1 can be understood as follows: decreasing $\kappa$ increases the effect of the last term in Eq. (1), leading to a bigger DAP at the soma, and is qualitatively the same as decreasing $g_{\mathrm{K}, \mathrm{d}}$. Decreasing $g_{\mathrm{K}, \mathrm{d}}$ was found in Ref. [2] to move the curve of saddle-node bifurcations of periodic orbits closer to the curve of saddle-node bifurcations of fixed points on a circle, such that they eventually meet at a codimension-two point. For $\kappa<\sim 0.35$, the neuron switches from quiescence to doublets as $I$ is increased, in a similar way to that shown in Ref. [2] for small $g_{\mathrm{K}, \mathrm{d}}$. As $\kappa$ is increased, the effect of the dendrite on the soma decreases, and since it was determined in Ref. [3] that slow cumulative inactivation of dendritic potassium is essential for ghostburster-type bursting, it is clear that for large enough $\kappa$, bursting will not occur. This explains the observation in Fig. 1 that for $\kappa>\sim 0.5$, only quiescent or tonic behavior is seen.

\subsection{Varying $g_{\mathrm{c}}$ (soma-dendrite coupling conductance)}

We now consider the effect of varying the conductance between the soma and dendrite, $g_{\mathrm{c}}$ (in Ref. [2], $g_{\mathrm{c}}$ was set to 1). In Fig. 2, we show a partial bifurcation set using $I$ and $g_{\mathrm{c}}$ as parameters. For $g_{\mathrm{c}}$ between about 0.2 and 1.7, the neuron moves from quiescence to tonic firing through a saddle-node bifurcation of fixed points on a circle (solid line) as $I$ is increased, and then to bursting through a saddle-node bifurcation of periodic orbits (dashed). For $g_{\mathrm{c}}>\sim 1.7$, or $<\sim 0.2$, only periodic firing occurs. This can be explained as follows. 


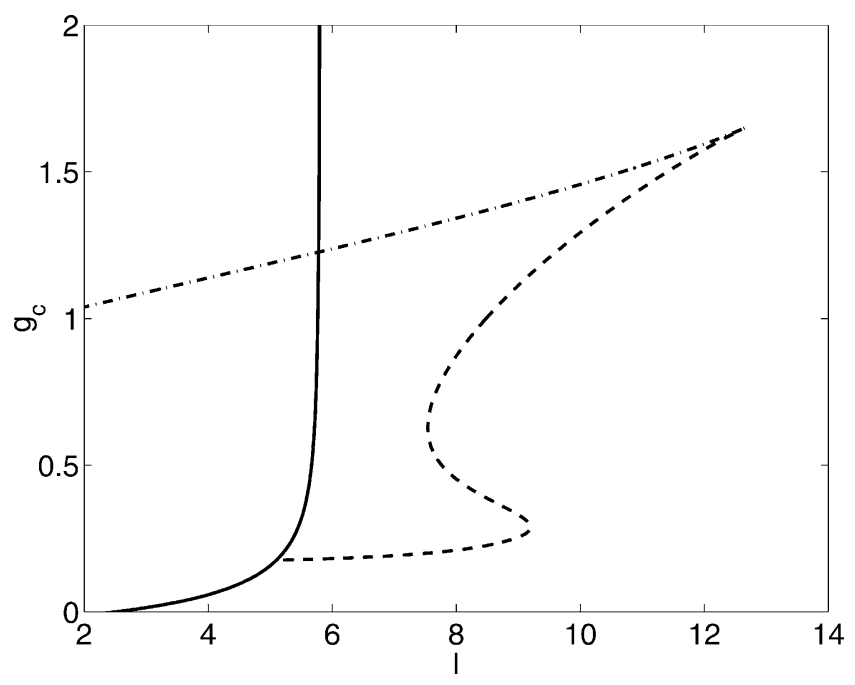

Fig. 2. Bifurcation set using $I$ and $g_{\mathrm{c}}$ as bifurcation parameters. The solid curve indicates a saddle-node bifurcation of fixed points on a circle, dashed is a saddle-node bifurcation of periodic orbits (one of which is stable), and the dashed-dotted is a saddle-node bifurcation of periodic orbits, both of which are unstable. $\kappa=0.4$.

For large $g_{\mathrm{c}}$, the voltages in the soma and dendrite track one another very closely. An essential ingredient for bursting is the dendritic to somatic current that causes the DAP, and specifically, the slow growth of the DAP. In this model, this current is purely a result of the different halfwidths of the somatic and dendritic action potentials - the somatic is normally shorter than the dendritic. If the somatic and dendritic voltages track one another closely, the effect of these different halfwidths, and thus the DAP, is removed. Hence there is no bursting for large $g_{\mathrm{c}}$. However, when $g_{\mathrm{c}}$ is small, the effective coupling between the soma and dendrite is also small. The soma is not capable of bursting by itself, so for small $g_{\mathrm{c}}$, the neuron moves from quiescence to tonic firing as $I$ is increased, in the same way as a single-compartment type I neuron [4].

\section{Conclusion}

We have investigated the effects of varying parameters related to the coupling between the soma and dendrite of a two-compartment model of a pyramidal cell that undergoes "ghostbursting" [2]. We have found that both parameters must have moderate values for bursting to occur. This can be explained in terms of the effects of changing these parameters on the known mechanism involved in bursting [2,3]. It is of interest to vary $\kappa$ because pyramidal cells in the ELL have been measured to have a wide range of $\kappa$ values, and furthermore, cells with smaller $\kappa$ are more likely to burst via soma-dendrite interactions [1], in agreement with our results. 


\section{References}

[1] J. Bastian, J. Nguyenkim, Dendritic modulation of burst-like firing in sensory neurons, J. Neurophysiol. 85 (2001) 10-22.

[2] B. Doiron, C. Laing, A. Longtin, L. Maler, Ghostbursting: a novel neuronal burst mechanism. J. Comput. Neurosci. 12 (2002) 5-25.

[3] B. Doiron, A. Longtin, R.W. Turner, L. Maler, Model of gamma frequency burst discharge generated by conditional backpropagation, J. Neurophysiol. 86 (2001) 1523-1545.

[4] B. Ermentrout, Type I membranes, phase resetting curves, and synchrony, Neural Comput. 8 (1996) 979-1001.

[5] J. Feng, G. Li, Behaviour of two-compartment models, Neurocomput. 38-40 (2001) 205-211.

[6] F. Gabbiani, W. Metzner, Encoding and processing of sensory information in neuronal spike trains, J. Expt. Biol. 202 (1999) 1267-1279.

[7] E.M. Izhikevich, Neural excitability, spiking, and bursting, Int. J. Bifn. Chaos. 10 (2000) 1171-1266.

[8] J. Keener, J. Sneyd, Mathematical Physiology, Springer, New York, 1998.

[9] A. Kepecs, X.-J. Wang, Analysis of complex bursting in cortical pyramidal neuron models, Neurocomput. 32-33 (2000) 181-187.

[10] N. Lemon, R. Turner, Conditional spike backpropagation generates burst discharge in a sensory neuron, J. Neurophysiol. 84 (2000) 1519-1530.

[11] J.E. Lisman, Bursts as units of neural information: making unreliable synapses reliable, Trends Neurosci. 20 (1997) 38-43.

[12] Z.F. Mainen, T.J. Sejnowski, Influence of dendritic structure on firing pattern in model neocortical neurons, Nature 382 (1996) 363-366.

[13] P.F. Pinsky, J. Rinzel, Intrinsic and network rhythmogenesis in a reduced Traub model for CA3 neurons, J. Comput. Neurosci. 1 (1994) 39-60.

[14] R.W. Turner, L. Maler, Oscillatory and burst discharge in the Apteronotid electrosensory lateral line lobe, J. Expt. Biol. 202 (1999) 1255-1265. 